\title{
Thyroid Hormone Resistance in a Preterm Infant with a Novel THRB Mutation
}

\author{
Joon Yeol Bae ${ }^{1}$, Dong Yeop Kim ${ }^{1}$, Young Dai Kwon ${ }^{1}$, Young Hwa Song ${ }^{1}$, Han Hyuk Lim² ${ }^{2}$ Hyung-Doo Park ${ }^{3}$, and Jae \\ Woo Lim ${ }^{1}$ \\ ${ }^{1}$ Department of Pediatrics, Konyang University College of Medicine, Daejeon, Korea \\ ${ }^{2}$ Department of Pediatrics, Chungnam National University College of Medicine, Daejeon, Korea \\ ${ }^{3}$ Department of Laboratory Medicine and Genetics, Samsung Medical Center, Sungkyunkwan University School of Medicine, Seoul, \\ Korea
}

\section{ABSTRACT}

Resistance to thyroid hormone (RTH) is a condition caused by a mutation in the thyroid hormone receptor gene. It is rarely reported in individuals with no family history of RTH or in premature infants, and its clinical presentation varies. In our case, a premature infant with no family history of thyroid diseases had a thyroid stimulating hormone level of $85.0 \mu \mathrm{IU} / \mathrm{mL}$ and free thyroxine level of $1.64 \mathrm{ng} / \mathrm{dL}$ on a thyroid function test. The patient also presented with clinical signs of hypothyroidism, including difficulties in feeding and weight gain. The patient was treated with levothyroxine; however, only free thyroxine and triiodothyronine levels increased without a decrease in thyroid-stimulating hormone levels. Taken together with thyroid gland hypertrophy observed on a previous ultrasound examination, RTH was suspected and the diagnosis was eventually made based on a genetic test. A de novo mutation in the thyroid hormone receptor $\beta$ gene in the infant was found that has not been previously reported. Other symptoms included tachycardia and pulmonary hypertension, but gradual improvement in the symptoms was observed after liothyronine administration. This report describes a case involving a premature infant with RTH and a de novo mutation, with no family history of thyroid disease.

Key Words: Thyroid hormone resistance syndrome; Infant, premature; Thyroid hormone receptors beta; Goiter; Triiodothyronine

\section{INTODUCTION}

Resistance to thyroid hormone (RTH) is a genetic disorder caused by a thyroid hormone receptor (THR) gene mutation, which results in a reduced response of THRs located on tissues $^{1-3)}$. RTH mostly occurs because of a mutation in thyroid hormone receptor beta (THRB); however, RTH caused by a mutation in the thyroid hormone receptor $\alpha$ gene (THRA) was also reported recently ${ }^{4}$.
Received: 27 August 2018

Revised: 13 March 2019

Accepted: 29 March 2019

Correspondence to: Jae Woo Lim

Department of Pediatrics, Konyang University Hospital, Konyang University College of Medicine, 158 Gwanjeodongro, Seo-gu, Daejeon 35365, Korea

Tel: +82-42-600-9230

Fax: +82-42-600-9090

E-mail: jwlim@kyuh.ac.kr

https://orcid.org/0000-0003-2001-0727
Copyright(c)

By Korean Society of Neonatology.

All right reserved.

This is an Open-Access article distributed under the terms of the Creative Commons Attribution Non-Commercial License (http://creativecommons.org/licenses/ by-nc/4.0), which permits unrestricted non-commercial use, distribution, and reproduction in any medium, provided the original work is properly cited. 
RTH is characterized by elevated free thyroxine (FT4) and triiodothyronine (T3) levels and normal or elevated thyroidstimulating hormone (TSH) levels; however, TSH levels decrease when a supraphysiologic dose of thyroid hormone is administered $^{1)}$. The clinical features of RTH are determined by the severity of hormone resistance. In most pediatric patients with RTH, symptoms of goiter, thyrotoxicosis, developmental disorders, and growth failure accompanied by delayed bone age are observed $^{1,2,5,6)}$.

RTH is typically inherited in an autosomal dominant pattern and thus is often identified in advance by taking the family history ${ }^{7}$. RTH affects approximately one in 40,000 newborns and is rarely diagnosed in premature infants ${ }^{8)}$. Here, we report a case involving a premature infant with RTH and a de novo mutation, with no family history of thyroid disease, together with tachycardia and pulmonary hypertension findings.

\section{CASE REPORT}

The female infant in our case was delivered naturally at a gestational age of 36 weeks and 1 day. Her mother was 42 years old and had an obstetric history of gravida 3, para 3, living 3, death 0 , abortion 0 . Specific family history of diseases including thyroid disease were absent. The mother gave birth after uncontrollable early pain and presence of oligohydramnios (amniotic fluid index of $3 \mathrm{~cm}$ ) on a prenatal examination. The delivery was performed rapidly. The infant had 1- and 5-minute Apgar scores of 4 and 7 points, respectively. Immediately after birth, the infant exhibited signs of cyanosis, meconium staining throughout the entire body, and poor activity. Cyanosis and poor activity were ameliorated after ambu-bagging. The infant weighed 2,160 g (3rd to 10th percentile) and was $43 \mathrm{~cm}$ in height (3rd to 10th percentile) with a head circumference of $31 \mathrm{~cm}$ (10th to 25 th percentile). Her blood pressure was $82 / 51 \mathrm{~mm} \mathrm{Hg}$; heart rate, 165 beats per minute; respiratory rate, 67 beats per minute; body temperature, $36.5^{\circ} \mathrm{C}$; and $\mathrm{SpO}_{2}, 90 \%$ on room air. Physical examination of the infant revealed chest retraction on breathing. Chest X-ray imaging results showed irregular opacity and decreased lung volume. Consequently, mechanical ventilation therapy was initiated and surfactant was used based on a diagnosis of meconium aspiration syndrome. Initial arterial blood gas analysis on $\mathrm{FiO}_{2}$ 0.5 revealed a $\mathrm{pH}$ of 7.35; $\mathrm{pCO}_{2}, 36 \mathrm{~mm} \mathrm{Hg}$; $\mathrm{pO}_{2}, 86.1 \mathrm{~mm} \mathrm{Hg}$; and $\mathrm{HCO}_{3}{ }^{-}, 20.3 \mathrm{mEq} / \mathrm{L}$. On the 2nd day of birth, extubation was performed and improvement in respiratory symptoms was observed, but tachypnea persisted. Non-invasive ventilation was applied for 9 days. At birth, heart rate increased to 180 beats per minute, and when she was irritable, her heart rate increased to 200 beats per minute. Echocardiography performed on day 4 of hospitalization showed pulmonary hypertension accompanied by severe right ventricular hypertrophy; hence, oral sildenafil was administered. Echocardiography performed on day 12 showed persistent cardiac hypertrophy and tricuspid regurgitation along with sinus tachycardia with a heart rate of 180 to 200 beats per minute; hence, additional oral atenolol was administered. Written informed consents were obtained.

A neonatal metabolic screening test performed at 5 days after birth and subsequent retest revealed an elevated TSH level but not thyroxine (T4) level in the infant. Eighteen days after birth, the infant had a TSH level of $75.3 \mu \mathrm{IU} / \mathrm{mL}$ (reference value, 0.5 to 10.0) and T4 level of $21.6 \mu \mathrm{g} / \mathrm{dL}$ (reference value, $>2.3$ ), indicating a gradual increase in the TSH level. Eighteen days after birth, goiter was suspected on physical examination. Accordingly, a serum test was performed on day 24 of hospitalization, which revealed a TSH level of $85.0 \mu \mathrm{IU} / \mathrm{mL}$ (reference value, 1.7 to 9.1), FT4 level of $1.64 \mathrm{ng} / \mathrm{dL}$ (reference value, 0.9 to 2.2), and T3 level of $636.63 \mathrm{ng} / \mathrm{dL}$ (reference value, 90 to 260). Based on these findings and continued difficulties in feeding and weight gain, levothyroxine (10 $\mu \mathrm{g} / \mathrm{kg} /$ day) was administered for suspected congenital hypothyroidism. Tests for thyroid disease antibodies (anti-thyroglobulin antibody, anti-microsomal antibody, and TSH receptor antibody) performed on day 24 of hospitalization revealed normal results and thyroid ultrasound imaging performed on day 25 of hospitalization showed hypertrophy of both

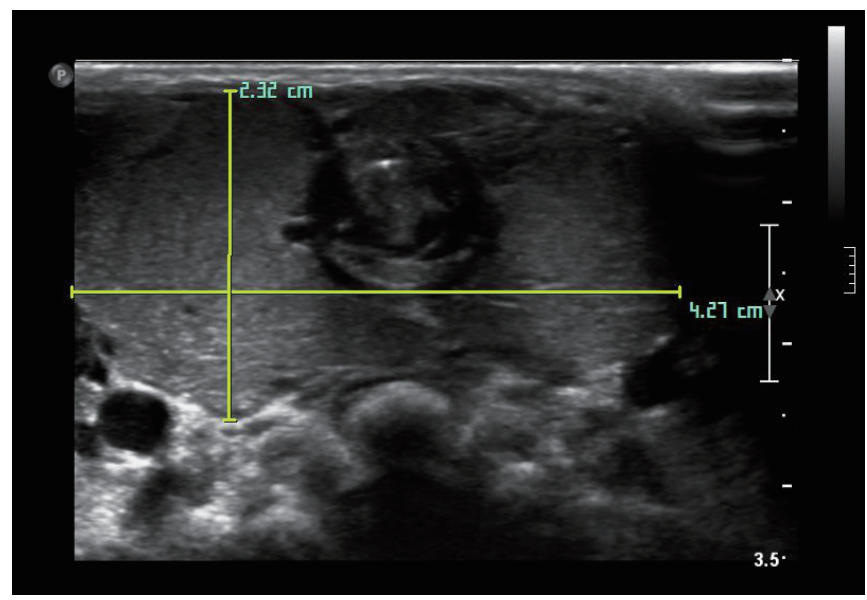

Figure 1. Thyroid ultrasonography. Hypertrophy of both thyroid glands $(2.3 \times 4.3 \mathrm{~cm})$. 
thyroid glands (Figure 1). Moreover, 99m technetium thyroid scanning performed on day 26 of hospitalization showed diffuse thyroid enlargement and increased thyroid uptake (Figure 2). The thyroid function test performed 1 week after levothyroxine administration revealed no improvement in the TSH level; hence, the levothyroxine dosage was increased to $15 \mu \mathrm{g} / \mathrm{kg} /$ day. Subsequently, the TSH level decreased while FT4 and T3 levels showed an abnormal increase, based on which levothyroxine administration was discontinued. The thyroid function test performed after discontinuation of levothyroxine administration revealed a TSH level of $85.0 \mu \mathrm{IU} / \mathrm{mL}$, FT4 level of $4.80 \mathrm{ng} / \mathrm{dL}$, and

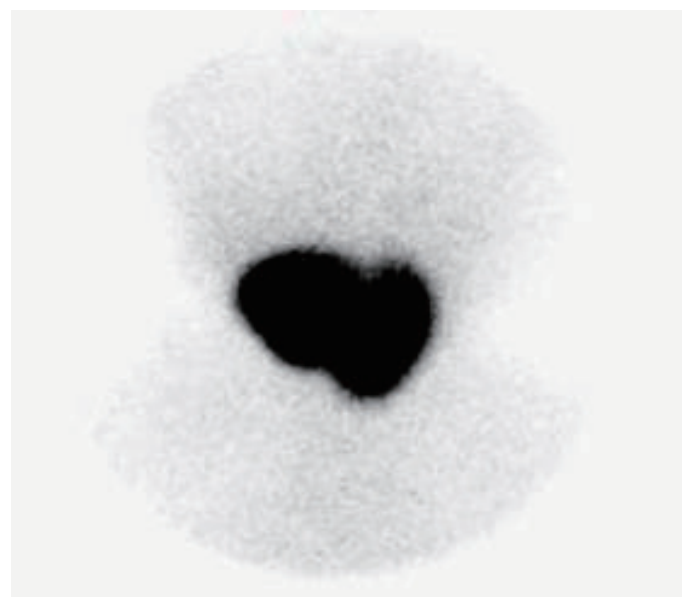

Figure 2. Technetium-99m thyroid scan showing homogeneously enlarged thyroid glands with markedly increased activity.
T3 level of $417.33 \mathrm{ng} / \mathrm{dL}$. Based on these findings, TSH secreting pituitary adenoma and RTH were suspected. Brain magnetic resonance imaging performed to eliminate the possibility of pituitary adenoma indicated no abnormalities. DNA sequencing was performed to test for RTH. Additionally, diagnostic auditory brainstem response confirmed severe sensorineural hearing loss in both ears. Direct sequencing of the infant's THRB gene performed on day 66 of hospitalization showed a heterozygous de novo mutation involving deletion of $\mathrm{G}$ at base 1320 [c.1320delG (p.His441Thrfs*2)] (Figure 3). Starting on day 72 of hospitalization, liothyronine (synthetic form of triiodothyronine, TETRONINE, Dalim Biotech, Hwaseong, Korea) was administered every alternate day to control the infant's goiter. The infant began to show signs of improvement a week later with a TSH level of $4.65 \mu \mathrm{IU} / \mathrm{mL}$, FT4 level of $4.72 \mathrm{ng} / \mathrm{dL}$, and T3 level of $214.45 \mathrm{ng} /$ dL (Table 1). Furthermore, goiter size decreased and heart rate returned to baseline levels. Thereafter, the patient was followed up on an outpatient basis and discharged. After discharge, the infant's parents underwent thyroid function and genetic testing, which revealed normal results.

The outpatient thyroid function test performed on day 104 after birth revealed that TSH $(12.77 \mu \mathrm{IU} / \mathrm{mL})$, FT4 (6.68 ng/ $\mathrm{dL}$ ), and T3 (649.34 ng/dL) levels were not controlled. Thus, the infant remained on liothyronine and is still being followed up. Echocardiography performed on day 115 after birth showed

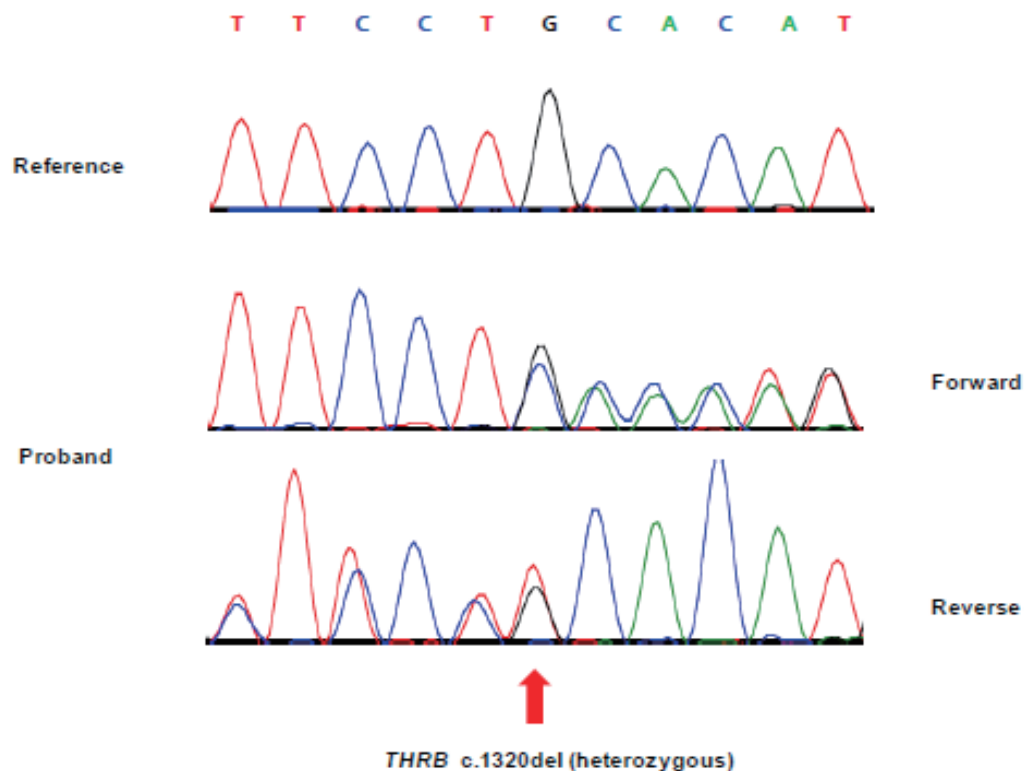

Figure 3. Direct sequencing of thyroid hormone receptor beta (THRB) in the patient. A heterozygous mutation (NM_000461.4:c. 1320delG(p.His441Thrfs*2)) was identified in the patient. 
Table 1. Thyroid Function Tests and Drug Regimen Medication of the Patient

\begin{tabular}{lccrrrrr}
\hline & \multicolumn{3}{c}{ Inpatient } & Outpatient \\
\hline PND $(\mathrm{d})$ & $18^{*}$ & 24 & 37 & 53 & 72 & 80 & 104 \\
TSH $(\mu \mathrm{IU} / \mathrm{mL})$ & 75.3 & 85.0 & 21.31 & 85.00 & 7.94 & 4.65 & 12.77 \\
FT4 (ng/dL) & & 1.64 & 8.83 & 4.80 & 8.23 & 4.72 & 6.68 \\
T3 (ng/dL) & & 636.63 & $1,959.10$ & 417.33 & 959.10 & 214.45 & \\
T4 $(\mu \mathrm{g} / \mathrm{dL})$ & 21.6 & & & Liothyronine start &
\end{tabular}

*Neonatal screening test: the third examination result.

Abbreviations: PND, postnatal day; TSH, thyroid-stimulating hormone; FT4, free thyroxine; T3, triiodothyronine; T4, thyroxine.

amelioration in right ventricular hypertrophy, tricuspid regurgitation, and tachycardia, and thus sildenafil and atenolol administration was discontinued. Currently, the infant shows mild developmental delay. Her goiter size increased again and thyroid hormone levels need to be controlled continuously.

\section{DISCUSSION}

This case is significant because RTH was diagnosed in a premature infant without a family history of thyroid disease. RTH is typically reported in children or adults and is rarely observed in neonates or premature infants ${ }^{9,10)}$. Additionally, RTH often occurs in individuals with a family history of the same disorder or other thyroid diseases ${ }^{11,12)}$. When the mother is confirmed to have the disease, a fetal DNA test may be performed to check for morbidity in the fetus ${ }^{13,14)}$. In our case, the infant was initially determined to have hypothyroidism with no family history of RTH, and a thyroid function test revealed an excessively high TSH level and normal FT4 level. RTH was suspected based on follow-up observations of subsequent thyroid function tests and the infant was eventually diagnosed with RTH based on genetic testing results. RTH is often misdiagnosed as hypothyroidism, hyperthyroidism, or thyroid cancer until a definitive diagnosis is established, resulting in ineffective drug or surgical treatment ${ }^{15)}$.

THRA and THRB encode THRs. Thyroid hormone receptors $\alpha 1$ (TR $\alpha 1)$ are typically located in the heart, bones, and brain; TR $\beta 1$ are located in the brain, liver, kidney, and thyroid gland; and TR $\beta 2$ are located in the pituitary, hypothalamus, retina, and inner ear ${ }^{1,2)}$. RTH is mostly associated with THRB mutations and clinical features of hypothyroidism may appear because of the resistance of peripheral tissues to thyroid hormone ${ }^{1)}$. In our case wherein a heterozygous mutation in THRB was confirmed, the infant presented with symptoms of hypothyroidism, including intrauterine growth retardation and difficulties in feeding and weight gain. Furthermore, symptoms of tachycardia and irrit ability were also observed, which are symptoms of thyrotoxicosis appearing from normal TR $\alpha$ stimulated by thyroid hormone. Because TR $\beta$ is found throughout the cochlea and plays an im portant role in hearing development, the sensorineural hearing loss in our case may be associated with this condition ${ }^{16)}$. More over, the symptoms of dyspnea and pulmonary hypertension in the infant shortly after birth and impaired thyroid function may be associated.

In a previously reported case, unlike in our case, RTH was identified in the mother. Abnormal thyroid hormone levels were found in the fetus, and the subsequent birth resulted in the infant being born with multiple organ failure and respiratory distress syndrome $^{13)}$. Additionally, hyperthyroidism increases catecholamine sensitivity and an overactive sympathetic nervous system increases pulmonary vasoconstriction and metabolism of intrinsic pulmonary vasodilators, leading to pulmonary hyperten $\operatorname{sion}^{17)}$.

Most cases of RTH remain untreated because they tend to be asymptomatic due to increased thyroid hormone secretion, which provides adequate compensation ${ }^{18)}$. However, when a patient has goiter and presents with various symptoms associated with RTH, as in the infant in our case, it is necessary to carefully monitor and administer thyroid hormone when required. Liothyronine is a synthetic form of T3 and metabolically active thyroid hormone that suppresses TSH. Liothyronine can be used to treat peripheral tissues in which the transition from T4 to T3 is impaired. Recent reports indicated that administering a supraphysiologic dose of T3 every alternate day effectively reduces goiter without inducing thyrotoxicosis ${ }^{19)}$. The infant in our case was also administered liothyronine every alternate day, after which hypertrophy of the thyroid glands disappeared and tachycardia improved 10 days after starting administration. However, because thyroid hormone 
levels continued to remain unstable, the patient currently remains on liothyronine and a follow up is being conducted. The clinical features of RTH are determined by the severity of hormone resistance. In partial RTH, the increase in the endogenous supply of thyroid hormone provides sufficient compensation; hence, no treatment is required, and its symptoms are mild or none. However, if the level of RTH is high, symptoms of thyroid hormone imbalance are more severe.

Another study on RTH treatment reported dextro-thyroxine administration in a patient with pituitary resistance to thyroid hormone (PRTH) to suppress the TSH, instead of liothyronine which was used in our case ${ }^{20)}$.

Because the clinical features of RTH are similar to those of hypothyroidism, a precise differential diagnosis is needed to ensure that the treatment is effective. If a neonate with a family history of RTH has high levels of TSH, FT4, and T3, RTH should be suspected first. Additionally, RTH should suspected if a neonate without a family history of RTH shows symptoms of hypothyroidism with high TSH, normal FT4, and high T3 levels, as in our case. Furthermore, if thyroid function tests following thyroid hormone administration yield unexpected results, it is important to discontinue drug administration and conduct a reevaluation.

\section{CONFLICT OF INTEREST}

No potential conflict of interest relevant to this article was reported.

\section{REFERENCES}

1. Olateju TO, Vanderpump MP. Thyroid hormone resistance. Ann Clin Biochem 2006;43(Pt 6):431-40.

2. Dumitrescu AM, Refetoff $S$. The syndromes of reduced sensitivity to thyroid hormone. Biochim Biophys Acta 2013;1830: 3987-4003.

3. Lee JH, Kim EY. Resistance to thyroid hormone due to a novel mutation of thyroid hormone receptor beta gene. Ann Pediatr Endocrinol Metab 2014;19:229-31.

4. Bochukova E, Schoenmakers N, Agostini M, Schoenmakers E, Rajanayagam O, Keogh JM, et al. A mutation in the thyroid hormone receptor alpha gene. N Engl J Med 2012;366:243-9.

5. Usala SJ, Bale AE, Gesundheit N, Weinberger C, Lash RW,
Wondisford FE, et al. Tight linkage between the syndrome of generalized thyroid hormone resistance and the human c-erbA beta gene. Mol Endocrinol 1988;2:1217-20.

6. Chatterjee VK, Gurnell M. Thyroid hormone resistance syndrome. In: Gale EAM, Wass JAH, Shalet SM, editors. Oxford textbook of endocrinology. London: Oxford University Press, 2002:339-49.

7. Yatsuga S, Hiromatsu Y, Sasaki S, Nakamura H, Katayama K, Nishioka J, et al. A two-day-old hyperthyroid neonate with thyroid hormone resistance born to a mother with well-conrolled Graves' disease: a case report. J Med Case Rep 2012;6:246.

8. Sambalingam D, Jyotsna Rao K. Neonatal "resistance to thyroid hormone (refetoff syndrome)" with novel THRB mutation. J Clin Neonatol 2017;6:273-5.

9. Refetoff S, Dumitrescu AM. Syndromes of reduced sensitivity to thyroid hormone: genetic defects in hormone receptors, cell transporters and deiodination. Best Pract Res Clin Endocrinol Metab 2007;21:277-305.

10. Pohlenz J, Weiss RE, Macchia PE, Pannain S, Lau IT, Ho H, et al. Five new families with resistance to thyroid hormone not caused by mutations in the thyroid hormone receptor beta gene. J Clin Endocrinol Metab 1999;84:3919-28.

11. Weiss RE, Weinberg M, Refetoff S. Identical mutations in unrelated families with generalized resistance to thyroid hormone occur in cytosine-guanine-rich areas of the thyroid hormone receptor beta gene. Analysis of 15 families. J Clin Invest 1993; 91:2408-15.

12. Kim JH, Park TS, Baek HS, Kim GH, Yoo HW, Park JH. A newly identified insertion mutation in the thyroid hormone receptorbeta gene in a Korean family with generalized thyroid hormone resistance. J Korean Med Sci 2007;22:560-3.

13. Asteria C, Rajanayagam O, Collingwood TN, Persani L, Romoli $\mathrm{R}$, Mannavola D, et al. Prenatal diagnosis of thyroid hormone resistance. J Clin Endocrinol Metab 1999;84:405-10.

14. Weiss RE, Dumitrescu A, Refetoff S. Approach to the patient with resistance to thyroid hormone and pregnancy. J Clin Endocrinol Metab 2010;95:3094-102.

15. Rivas AM, Lado-Abeal J. Thyroid hormone resistance and its management. Proc (Bayl Univ Med Cent) 2016;29:209-11.

16. Brucker-Davis F, Skarulis MC, Pikus A, Ishizawar D, Mastroianni MA, Koby M, et al. Prevalence and mechanisms of hearing loss in patients with resistance to thyroid hormone. J Clin Endo. crinol Metab 1996;81:2768-72.

17. Zuhur SS, Baykiz D, Kara SP, Sahin E, Kuzu I, Elbuken G. Relationship among pulmonary hypertension, autoimmunity, thyroid hormones and dyspnea in patients with hyperthyroidism. Am J Med Sci 2017;353:374-80.

18. Weiss RE, Refetoff S. Treatment of resistance to thyroid hormone: primum non nocere. J Clin Endocrinol Metab 1999;84: 
401-4.

19. Maruo Y, Mori A, Morioka Y, Sawai C, Mimura Y, Matui K, et al. Successful every-other-day liothyronine therapy for severe resistance to thyroid hormone beta with a novel THRB mutation; case report. BMC Endocr Disord 2016;16:1.
20. Dorey F, Strauch G, Gayno JP. Thyrotoxicosis due to pituitary resistance to thyroid hormones. Successful control with D thyroxine: a study in three patients. Clin Endocrinol (Oxf) 1990; 32:221-8. 